\title{
Perioperative Protocols in Colorectal Surgery
}

\author{
Mia DeBarros, MD $^{1} \quad$ Scott R. Steele, $M^{1}$ \\ ${ }^{1}$ Department of Surgery, Madigan Healthcare System, Tacoma, \\ Washington \\ Clin Colon Rectal Surg 2013;26:139-145.
}

\begin{abstract}
Address for correspondence Scott R. Steele, MD, Department of Surgery, Madigan Healthcare System, 9040a Fitzsimmons Dr, Tacoma, WA 98431 (e-mail: harkersteele@me.com).
\end{abstract}

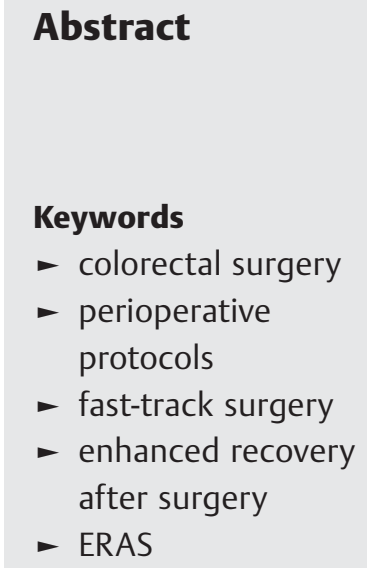

The reduction in health-care expenditures and more efficient use of medical resources are now overriding health policy priorities with the two-pronged goal of improving patient outcomes while decreasing overall cost. Current reports show colorectal surgery accounting for $25 \%$ of all operative complications and an average length of stay of 8 to 12 days for a standard elective colon resection. To combat this, Kehlet and colleagues introduced a concept of enhanced recovery after surgery (ERAS) or fast-track pathways, with the goal of using current evidence and multimodal therapies to decrease surgical stress, enhance postoperative recovery, and reduce length of stay. The benefits, safety, and cost-effectiveness of fast-track protocols are validated in multiple randomized controlled trials. In this review, the authors focus on the evidence regarding fast-track pathways, use of minimally invasive surgery and its role in fast-track pathways, newer perioperative interventions, and future directions.
Objectives: On completion of this article, the reader should be able to recognize that fast-track protocols are safe and costeffective, while improving overall patient outcomes by reducing the surgical stress response; summarize the components of fast-track surgery by each perioperative phase; understand the role of minimally invasive surgery in colon and rectal resections and perioperative adjuncts; and outline future directions for fast-track surgery.

In 2010, health-care expenditures in the United States neared $\$ 2.6$ trillion, 10 times the amount spent in $1980 .^{1}$ Current projections show national health expenditures continuing to increase and account for $20 \%$ of the gross domestic product by $2020 .^{2}$ The reduction in health-care expenditures and more efficient use of medical resources is now an overriding health policy priority with the two-pronged goal of improving patient outcomes while decreasing overall cost. This is especially relevant in the field of colorectal surgery because of a disproportionately higher complication rate and longer length of stay when compared with other operative procedures. Current reports show colorectal surgery accounting for $25 \%$ of all operative complications and an average length of stay of 8 to 12 days for a standard elective colon resection. ${ }^{3-6}$

During the last several decades, there has been growing recognition that surgical stress caused by major surgery results in derangements in organ function and subsequently increases postoperative morbidity. To combat this, Kehlet introduced a concept of enhanced recovery after surgery (ERAS) or fast-track pathways, with the goal of using current evidence and multimodal therapies to reduce surgical stress and enhance postoperative recovery. This was achieved by asking the simple question: Why is the patient in the hospital today? ${ }^{7}$ The pathway elements are perioperative care interventions that focus on anesthesia, analgesia, reduction of surgical stress (both endocrine-metabolic and inflammatory responses), goal-directed fluid therapy, the prevention of nausea and ileus (return of bowel function), thromboembolic prophylaxis, minimally invasive techniques, nutrition, and early mobilization. ${ }^{8}$

The benefits and safety of fast-track protocols (FTPs) are validated in multiple randomized controlled trials. ${ }^{9}$ Patients in these studies had faster return of bowel function, shorter length of hospitalization, and decreased complication rates. These findings were confirmed by several meta-analyses and a recent Cochrane Review. ${ }^{10-13}$ The most recent meta-analysis included 7 randomized control trials with 852 patients. Lv et al found patients randomized to FTP care had a significantly decreased length of stay (mean difference: -1.88 ; $95 \%$ confidence interval [CI], -2.91 to $0.86, p=0.0003$ ) and overall rate of complications (relative risk $[\mathrm{RR}]=0.69 ; 95 \% \mathrm{CI}, 0.51-0.93$ ),
Issue Theme Perioperative Management; Guest Editor, Sharon L. Stein, MD, FACS
Copyright @ 2013 by Thieme Medical Publishers, Inc., 333 Seventh Avenue, New York, NY 10001, USA. Tel: +1(212) 584-4662.
DOI http://dx.doi.org/ 10.1055/s-0033-1351128. ISSN 1531-0043. 
$p=0.01)$ without differences in mortality $(\mathrm{RR}=1.02 ; 95 \%$ $\mathrm{CI}=0.40-2.57, p=0.97) .^{13}$ Initial reports demonstrated higher readmission rates in two small studies (likely secondary to the earlier discharge); however, in several subsequent meta-analyses, there was no significant difference in readmission rates between conventional care and FTP patients. ${ }^{10-15}$

Regarding concerns of higher cost, multiple studies have found FTPs to be cost-efficient, even including those that use laparoscopic surgery. Laparoscopic surgery is more expensive than open surgery secondary to disposable instruments, additional operative time, and mastery of the learning curve. ${ }^{16-18}$ However, overall costs for laparoscopy are offset by reduction in length of stay, inpatient resources, and decreased complications. Delaney and colleagues reported implementation of an FTP for minimally invasive surgery at a single institution that required no additional personnel or expense. ${ }^{19} \mathrm{~A}$ cost analysis in New Zealand found an initial implementation cost of $\$ 102,000$ was offset by a reduction in postoperative resource utilization and an overall cost savings of $\$ 6,900$ per patient. ${ }^{20}$

This review focuses on the evidence regarding fast-track pathways, use of minimally invasive surgery and its role in fast-track pathways, newer perioperative interventions, and future directions. Some brief overviews of the individual components are reviewed here with more detailed evidence regarding each component found elsewhere within this volume.

\section{Implementing an ERAS Pathway}

Fast-track surgery is a multimodal effort to improve patient outcomes and decrease cost of care. As such, implementation of fast-track surgery pathway requires a multimodal approach involving the entire patient-care team. A fast-track surgery team includes a team leader, anesthetists, nurses, and ancillary staff such as physical therapists, enterostomal therapists, and social workers-all trained in fast-track surgery principles. ${ }^{21}$

Despite substantial evidence in the literature on the benefit and cost savings of fast-track surgery, implementation of a FTP is challenging. Several series have shown that the transition from concept to wide clinical practice is delayed. ${ }^{19,22-25}$ In 2010, a Web-based survey of 407 general and colorectal surgeons found that only $30 \%$ of the surgeons practiced in hospitals that had implemented an FTP. ${ }^{26}$ Internal barriers include lack of awareness of current evidencebased literature regarding benefits of fast-track surgery, disagreement with current findings, or belief that a particular hospital or institution cannot support fast-track surgery protocols. External barriers include a lack of support from staff (hospital administration, nurses, and physicians), inability to collect and maintain outcome data, lack of expertise in FTPs, financial concerns regarding cost of implementation and maintenance of FTP, and insufficient number of support staff. ${ }^{8,27}$ The key to overcome many of these barriers is having the senior clinical and administrative leadership buy-in to fast-track surgery and the protocols required to make it successful. ${ }^{19}$ In addition, acceptance by the patient and his or her support system is mandatory. Education and communication of each component of fast-track pathway begins at the preoperative visit and is performed in verbal and written communications in simple, plain language. As the patient enters different phases of fast-track pathway, goals such as early ambulation, oral intake, pain control, and other discharge criteria are reiterated by all members of the team. A successful FTP hinges on patient and provider compliance, with the postoperative phase being the most critical in decreasing length of stay. ${ }^{28}$

\section{Components of Fast-Track Protocol}

\section{The Preoperative Phase}

FTP interventions begin with proper patient selection involving a thorough history and physical examination with all organ systems carefully reviewed to determine whether a patient requires preoperative optimization. Patients with chronic obstructive pulmonary disease, coronary artery disease, and diabetes mellitus may require additional workup and treatment before proceeding with surgery. ${ }^{29}$ Ideal patients for the FTP are healthy individuals who have an American Society of Anesthesiologist (ASA) score of 1 or 2, whereas typically only a select group of patients with ASA score of 3 are optimal candidates. ${ }^{30}$ Commonly reported contraindications for FTP include malnourishment (greater than $10 \%$ weight loss), immobility or minimally immobile, active alcohol abuse (more than 5 drinks per day) or dependence, poorly controlled psychiatric disorders or lack of social support, and inability to follow-up for postoperative visits or complications. ${ }^{29,30}$ Patients with active alcohol abuse or dependence should be abstinent at least 4 weeks prior to surgery to decrease postoperative morbidity. ${ }^{31,32}$ Active tobacco abusers are strongly encouraged to quit at least 4 weeks prior to surgery to prevent respiratory complications, although an optimal cessation period to decrease immune response and improve wound healing has yet to be determined. ${ }^{33-35}$ The type of procedure being performed should also be a factor in whether FTP is utilized. Emergent procedures for ischemia, obstruction, or perforation and difficult procedures that require extensive dissection or lysis of adhesions are likely best managed by the standard postoperative protocol..$^{30}$ The planned surgical procedure and FTP including risks and benefits are discussed at this time with appropriate FTP patients. ${ }^{30}$

Perioperative $\beta$-blockade has been extensively studied in standard care perioperative protocols. Current recommendations are to continue $\beta$-blockade in patients with known coronary artery disease already on this therapy or those with preoperative cardiac ischemia or cardiac symptoms scheduled to undergo high-risk surgery. When the decision is made to initiate $\beta$-blockade therapy, it is started several weeks prior to surgery and titrated to "appropriate and individual" heart rate and blood pressure. Patients who continue or are placed on $\beta$-blockade therapy require close monitoring for bradycardiac or hypotensive episodes in the postoperative period. ${ }^{36,37}$ 
Preoperative mechanical bowel preparation (MBP) was historically considered standard procedure on the basis of the belief that it reduced infection rates and anastomotic dehiscence by decreasing fecal and bacterial load. A recent Cochrane Review examined patients undergoing elective colon or rectal surgeries and found no evidence of clinical benefit in prevention of anastomotic leaks or wound infections. In elective extraperitoneal rectal surgery with restoration of continuity and patients undergoing laparoscopic resection, the authors concluded that further research is required. ${ }^{38}$ Recent guidelines from the Society of Alimentary Gastrointestinal Endoscopic Surgeons recommend MBP in laparoscopic surgeries to improve bowel manipulation and prepare for intraoperative colonoscopy if anastomotic visualization or lesion localization is required. In lower rectal resections or rectal resections with planned proximal diversion after resection and anastomosis, MBP is also recommended. ${ }^{39,40}$ Currently, there are no studies that specifically address MBP in an FTP.

\section{The Intraoperative Phase}

\section{The Pathophysiology of Surgical Stress}

The surgical stress response is thought to be a conserved cellular defense mechanism occurring as a result of surgical trauma. ${ }^{41,42}$ This causes changes in neural, endocrine, and metabolic systems with a shift toward catabolism, activation of sympathetic nervous system, and release of catecholamines. When surgical injury is induced, afferent neuronal impulses are stimulated at the site of injury and travel up sensory nerve roots through the dorsal horn of the spinal cord to the medulla and hypothalamus. There is a subsequent release of hypothalamic stimulating hormones and increased secretion of pituitary hormones, adrenocorticotropic hormone, antidiuretic hormone, and growth hormones. ${ }^{43}$ Increased secretion of these hormones shifts the body into a hyperdynamic and catabolic state. This results in increased oxygen and cardiac demand, decreased pulmonary function, pain, gastrointestinal (GI) side effects such as postoperative nausea and vomiting (PONV) and ileus, derangements of coagulation favoring a prothrombotic state, and immunosuppression. ${ }^{7}$ Tissue injury also causes activation of the inflammatory response with local and systemic effects. This is primarily mediated by cytokines, with an imbalance of proinflammatory (interleukin- [IL-] 1, IL-6, and tumor necrosis factor- $\alpha$ ) and anti-inflammatory cytokines, with the magnitude of the stress response related to the degree of surgical trauma. ${ }^{7,44,45}$ Proinflammatory cytokines also activate the hypothalamus-pituitary-adrenal axis. ${ }^{44}$

The overall goal of intraoperative components in fasttrack surgery is attenuation of surgical stress response. This is achieved either through blockade of inflammatory or endocrine-metabolic responses. Standard intraoperative techniques to minimize or block these responses include prevention of hypothermia, appropriate antibiotic prophylaxis, and avoidance of blood transfusions. FTP interventions also include neuraxial blockade and minimally invasive surgery.

\section{Neuraxial Blockade and Preemptive Analgesia}

Neuraxial blockade is an anesthetic technique providing optimal pain control by delivering local and opioid analgesia directly to the dorsal horn of the spinal cord and decreasing surgical stress response by blocking afferent neural impulses from the site of surgical injury to the hypothalamus and anterior pituitary gland. ${ }^{45,46}$ Epidural catheters have been shown to be superior to intravenous opioids in postoperative pain control in both open and laparoscopic surgery. ${ }^{47}$ There is also benefit shown in reduction of postoperative ileus (POI). ${ }^{48}$ Yet, in the FTP setting, some studies did not find a benefit to use epidural anesthesia.

Other techniques for preemptive analgesia include systemic lidocaine or transabdominal peritoneal block. Systemic lidocaine is generally administered intravenously with bolus followed by continuous infusion to attenuate surgical stress response and prevention of POI. A double-blind, randomized, placebo controlled trial found systemic lidocaine infusions significantly decreased time to GI tract recovery ( 8 hours earlier than control, $p<0.05$ ) and length of stay ( 7 days in lidocaine group versus 8 days in control group, $p=0.004$ ) and attenuated elevated plasma levels of inflammatory cytokines. ${ }^{49}$

The transversus abdominis plane (TAP) block was first reported by Rafi in 2001 and has since been modified several times. ${ }^{50}$ Current technique involves infusion of local anesthetics into the neurovascular plane between the transversus abdominis muscle and the internal oblique muscle via the lumbar triangle of Petit. This regional anesthetic technique is capable of blocking the lower intercostals (T7-11), iliohypogastrics, and ilioinguinal nerves. A prospective randomized trial validated its ability to decrease postoperative opioid requirements by $70 \%$ in 34 patients undergoing open elective colorectal surgeries. ${ }^{51}$ Although TAP blocks hold much promise in reduction of postoperative pain, opioid usage, and POI, more prospective randomized trials are needed to validate the above studies' findings.

\section{Minimally Invasive Surgery}

Laparoscopy in colorectal surgery improves postoperative outcomes when compared with an open approach, largely through decreased pulmonary complications, smaller incisions resulting in decreased pain and opioid use, reduction in POI, and overall decreased average length of stay by 2 to 3 days compared with conventional open surgery. ${ }^{16,52,53}$ Recent Cochrane Reviews confirmed short-term benefits of laparoscopic colorectal surgery and long-term outcomes of laparoscopic oncological resections. ${ }^{54,55}$ Despite these findings, there is a slower rate of integration within the field of colorectal surgery, with only a third of all elective colectomies in the United States performed laparoscopically.

With introduction of FTPs into colorectal surgery, there is increasing interest in further optimizing reduction of surgical stress, and its negative effects, by adopting minimally invasive techniques. Single-center studies demonstrate a shorter length of stay (3.7-4.1 d) with no increase in readmission or complication rates. ${ }^{56-58}$ Poon et al found decreased rate of POI when evaluating colorectal cancer patients undergoing 
laparoscopic colectomy with FTP, but did not find a statistically significant difference in length of stay, complication, or readmission rates. ${ }^{59}$

There are also conflicting findings about cost-effectiveness. An English study reported a significant reduction in length of stay compared with open procedures (5.2 vs. 7.4 d), but higher upfront operative costs ( $\$ 4,016$ vs. $\$ 2,781$ ), which were offset by decreased length of stay, readmission rates, and follow-up costs; the laparoscopic/FTP group actually saved $\$ 517$ ( $\$ 9,405$ vs. $\$ 9,922) .{ }^{60}$ The traditional open, open fast-track recovery and laparoscopic fast-track multimodal management (TAPAS) study is an ongoing prospective cohort study evaluating cost-effectiveness of patients undergoing resection for colon cancer using laparoscopic techniques and FTPs in three treatment arms (open surgery with standard treatment, open surgery with fast-track treatment, and laparoscopic surgery with fast-track treatment). Primary outcomes are direct medical costs and indirect nonmedical costs to direct the future investment of minimally invasive techniques, FTPs, or a combination of both. ${ }^{61}$

To determine what the optimal combination of perioperative care is, several multicenter trials have been undertaken. The laparoscopy and/or fast-track multimodal management versus standard care (LAFA) study randomized patients with colon cancer to four different arms: laparoscopic colectomy with fast-track care, laparoscopic colectomy with standard care, open colectomy with fast-track care, or open colectomy with standard care. The shortest length of stay occurred in the laparoscopic/FTP group, with laparoscopy the only independent predictive factor to reduce length of stay and morbidity after regression analysis. ${ }^{62}$ Further multivariate and univariate analysis of baseline characteristics and fasttrack elements demonstrated early enteral feeding, early mobilization, laparoscopic surgery, and female sex as independent determinants of early recovery. ${ }^{63}$ The enhanced recovery open versus laparoscopic (EnROL) trial is an ongoing multicenter, randomized control trial that stratifies patients to open or laparoscopic colorectal cancer surgery with both groups receiving fast-track care. This trial seeks to determine whether laparoscopic surgery improves outcomes compared with open surgery, even when fast-track care is used. This trial differs from the LAFA study by including patients undergoing resection for rectal carcinoma and excluding benign colorectal disease to capture a sicker, older patient population who likely have a higher complication rate and thus highlight potential benefits of laparoscopy and fasttrack care. ${ }^{64}$

The use of minimally invasive surgery has also been explored in rectal surgery for both benign and malignant disease. ${ }^{65}$ The most recent recommendations published by the ERAS Society strongly recommend laparoscopy in an FTP for resection of benign disease in the hands of an experienced surgeon, but not for malignant disease unless in the setting of a trial until a final consensus is reported from ongoing trials. ${ }^{66}$

Minimally invasive surgery is another fast-track surgery technique to decrease the surgical stress response and improve patient outcomes. Although more costly upfront, this is offset by decreased length of stay, inpatient resources, and postoperative morbidity. There are multiple studies to support its use in colonic resections; however, in rectal resections current evidence is limited at this time.

\section{The Postoperative Phase}

\section{Recovery of Gastrointestinal Function}

POI is a dreaded, but common complication after major abdominal surgery. POI is defined as a "temporary impairment of GI function" and characterized by "bowel distension and vomiting and delay in oral feeding and increased postoperative pain. ${ }^{52}$ The exact etiology of $\mathrm{POI}$ is unknown, but believed to be multifactorial with activation of sympathetic reflexes at spinal and local levels and the inflammatory response. Open surgery and increased bowel manipulation may contribute to POI. Laparoscopy, as part of the FTP, is believed to reduce surgical trauma and the inflammatory response, thus avoiding the development of POI; however, a Cochrane Review found laparoscopy can reduce but not prevent POI. ${ }^{55}$ Cost of POI management is staggering with higher mean costs and length of stay with an overall cost in the United States ranging from $\$ 750$ million to $\$ 1.46$ billion dollars. ${ }^{67-70}$ FTPs attempt to lower incidence of POI by employing multimodal therapies. Current protocols include minimally invasive surgery, thoracic epidural analgesia, avoidance of routine nasogastric intubation, and early enteral feeding.

\section{Judicious Pain Management}

The postoperative phase is the most critical phase in increasing patient compliance and decreasing length of stay. To that end, judicious and effective postoperative pain management is crucial. Systemic opioids are a traditional part of pain management, with excellent results seen with patient-controlled analgesia use; however, they are not without their side effects including worsening of POI. The use of continuous epidural analgesia, lidocaine infusions, and TAP blocks may represent methods to deliver effective pain control and attenuate the surgical stress response and should be utilized in fast-track surgery protocols.

Additional options for multimodality postoperative pain management are discussed in the last article in this issue of Clinics in Colon and Rectal Surgery. These include regional, local infiltration (liposomal bupivacaine), and nonopioid systemic analgesia (intravenous acetaminophen and nonsteroidal anti-inflammatory drugs). ${ }^{71}$

\section{Future Directions}

Since its inception in the mid-1990s, FTP continues to undergo refinement in an effort to improve patient outcomes. Preoperative risk assessment is a cornerstone of all successful surgical interventions. The tools and matrices that allow for careful assessment and further optimization are based on conventional surgical protocols. As older and sicker patients undergo surgery, further studies of these patients within an FTP are needed to reassess perioperative risk. ${ }^{72}$ 
Table 1 Laparoscopic colectomy with fast-track protocol

\begin{tabular}{|c|c|c|c|c|c|c|}
\hline Author & Year & $\begin{array}{l}\text { Total number } \\
\text { of patients }\end{array}$ & $\begin{array}{l}\text { No. of laparoscopic } \\
\text { with FTP patients }\end{array}$ & $\begin{array}{l}\text { Mean length } \\
\text { of stay (days) }\end{array}$ & $\begin{array}{l}\text { Complication } \\
\text { rate (\%) }\end{array}$ & $\begin{array}{l}\text { Readmission } \\
\text { rate (\%) }\end{array}$ \\
\hline Bardem et $\mathrm{al}^{73}$ & 2000 & 50 & 50 & 2.5 & 0.25 & 0.05 \\
\hline Basse et $\mathrm{al}^{74}$ & 2005 & 60 & 30 & 3.8 & 0.26 & 0.2 \\
\hline King et $\mathrm{al}^{60}$ & 2006 & 58 & 41 & 5.2 & 0.15 & 0.05 \\
\hline Delaney et $\mathrm{al}^{53}$ & 2008 & 118 & 118 & $3.7 \pm 3.7$ & 20.3 & 8.5 \\
\hline $\begin{array}{l}\text { Reurings et al } \\
\text { (TAPAs Trial) }^{61}\end{array}$ & 2010 & 120 & $40^{a}$ & b & b & b \\
\hline Vlug et al (LAFA Trial) ${ }^{62}$ & 2011 & 427 & 100 & $5(4-8)^{c}$ & $34^{d}$ & $6^{d}$ \\
\hline Poon et $\mathrm{al}^{59}$ & 2011 & 180 & 96 & $4(2-23)^{c}$ & $12.5^{d}$ & $5^{d}$ \\
\hline Delaney et $\mathrm{al}^{57}$ & 2012 & 1000 & 1000 & $4.1 \pm 3.2$ & 20 & 6 \\
\hline $\begin{array}{l}\text { Kennedy et al } \\
\text { (EnROL Trial) }^{64}\end{array}$ & 2012 & 202 & $133^{a}$ & b & b & b \\
\hline
\end{tabular}

aPlanned sample size.

bongoing trial.

'Median length of stay.

${ }^{\mathrm{d}}$ Not significant, $p>0.05$.

The overall reduction in surgical stress is a priority in FTP. Attenuation of this response is an active area of research. Research into the efficacy of laparoscopy in fully implemented FTPs is ongoing with the EnROL trial and results are expected in the next year (-Table 1). ${ }^{64}$ Natural orifice transluminal endoscopic surgery and single-incision laparoscopic surgery are the latest techniques in minimally invasive surgery, but their implementation and benefit in FTP has yet to be investigated. ${ }^{72}$

\section{Conclusion}

Fast-track surgery uses current evidence and multimodal therapies to reduce surgical stress and enhance postoperative recovery by asking the simple question: Why is the patient in the hospital today? ${ }^{7}$ Whereas FTPs may encompass different individual components, each with varying reported degrees of success, their overall usefulness is likely a reflection of the entire protocol. What seems clear is that FTPs are safe and cost-effective, and result in a reduction in both length of stay and postoperative morbidity and mortality. A successful FTP is a multidisciplinary endeavor that requires support from all members of the team, including the management of patients' expectations. Although full implementation of all components continues to be a challenge, it is important to understand the evidence behind their success. Many programs continue to exclude proven evidence-based components such as proper patient information, utilization of neuraxial anesthesia, nonopioid analgesia, or early enteral feeding. ${ }^{72}$ These tools are proven to improve postoperative mobility and decrease risk of POI. Although gains in the reduction of surgical stress, patient outcome, and cost have been made, continuing reassessment and research is needed to further refine and improve upon the components of the fast-track surgery protocol.

\section{Disclaimers}

The views expressed are those of the author(s) and do not reflect the official policy of the Department of the Army, the Department of Defense, or the US Government.

\section{References}

1 Centers for Medicare and Medicaid Services, Office of the Actuary, National Health Statistics Group. U.S. Department of Commerce, Bureau of Economic Analysis; and U.S. Bureau of the Census. National Healthcare Expenditures Data. Baltimore, MD: U.S. Department of Commerce and U.S. Bureau of the Census; 2012

2 The Commonwealth Fund Commission on a High Performance Health System. The path to a high performance health system: a 2020 vision and the policies to pave the way. Available at: http:// www.commonwealthfund.org. Accessed October 10, 2012

3 Schilling PL, Dimick JB, Birkmeyer JD. Prioritizing quality improvement in general surgery. J Am Coll Surg 2008;207:698-704

4 Longo WE, Virgo KS, Johnson FE, et al. Risk factors for morbidity and mortality after colectomy for colon cancer. Dis Colon Rectum 2000;43(1):83-91

5 Adamina M, Kehlet H, Tomlinson GA, Senagore AJ, Delaney CP. Enhanced recovery pathways optimize health outcomes and resource utilization: a meta-analysis of randomized controlled trials in colorectal surgery. Surgery 2011;149(6):830-840

6 Kehlet H. Fast-track colorectal surgery. Lancet 2008;371:791-793

7 Kehlet H. Multimodal approach to control postoperative pathophysiology and rehabilitation. Br J Anaesth 1997;78(5):606-617

8 Kehlet H, Wilmore DW. Evidence-based surgical care and the evolution of fast-track surgery. Ann Surg 2008;248(2):189-198

9 Lassen K, Soop M, Nygren J, et al; Enhanced Recovery After Surgery (ERAS) Group. Consensus review of optimal perioperative care in colorectal surgery: Enhanced Recovery After Surgery (ERAS) Group recommendations. Arch Surg 2009;144(10):961-969

10 Wind J, Polle SW, Fung Kon Jin PH, et al; Laparoscopy and/or Fast Track Multimodal Management Versus Standard Care (LAFA) Study Group; Enhanced Recovery after Surgery (ERAS) Group. Systematic review of enhanced recovery programmes in colonic surgery. Br J Surg 2006;93(7):800-809 
11 Walter CJ, Collin J, Dumville JC, Drew PJ, Monson JR. Enhanced recovery in colorectal resections: a systematic review and metaanalysis. Colorectal Dis 2009;11(4):344-353

12 Spanjersberg WR, Reurings J, Keus F, van Laarhoven CJ. Fast track surgery versus conventional recovery strategies for colorectal surgery. Cochrane Database Syst Rev 2011;(2):CD007635

$13 \mathrm{Lv}$ L, Shao YF, Zhou YB. The enhanced recovery after surgery (ERAS) pathway for patients undergoing colorectal surgery: an update of meta-analysis of randomized controlled trials. Int J Colorectal Dis 2012;27(12):1549-1554

14 Khoo CK, Vickery CJ, Forsyth N, Vinall NS, Eyre-Brook IA. A prospective randomized controlled trial of multimodal perioperative management protocol in patients undergoing elective colorectal resection for cancer. Ann Surg 2007;245(6):867-872

15 Hjort Jakobsen D, Sonne E, Basse L, Bisgaard T, Kehlet H. Convalescence after colonic resection with fast-track versus conventional care. Scand J Surg 2004;93(1):24-28

16 Delaney CP, Kiran RP, Senagore AJ, Brady K, Fazio VW. Casematched comparison of clinical and financial outcome after laparoscopic or open colorectal surgery. Ann Surg 2003;238(1):67-72

17 Basse L, Hjort Jakobsen D, Billesbølle P, Werner M, Kehlet H. A clinical pathway to accelerate recovery after colonic resection. Ann Surg 2000;232(1):51-57

18 Wind J, Hofland J, Preckel B, Hollmann MW, Bossuyt PM, Gouma DJ. Perioperative strategy in colonic surgery: LAparoscopy and or FAst Track Multimodal Management versus Standard Care (LAFA Trial). BMC Surg 2006;6:6

19 Keller D, Delaney CP. The role of enhanced recovery pathways in the setting of minimally invasive colorectal surgery. Seminars in Colorectal Surgery 2012; In press

20 Sammour T, Zargar-Shoshtari K, Bhat A, Kahokehr A, Hill AG. A programme of Enhanced Recovery After Surgery (ERAS) is a costeffective intervention in elective colonic surgery. $\mathrm{N} \mathrm{Z} \mathrm{Med} \mathrm{J}$ 2010;123(1319):61-70

21 Stein SL, Delaney CP. Standardized fast track protocols or enhanced recovery pathways. In: Beck DE, Roberts PL, Saclarides TJ, Senagore AJ, Stamos MJ, Wexner SD, eds. The ASCRS Textbook of Colon and Rectal Surgery. 2nd ed. Heidelberg, Germany: Springer; 2011

22 Kehlet H, Büchler MW, Beart RWJ Jr, Billingham RP, Williamson R. Care after colonic operation-is it evidence-based? Results from a multinational survey in Europe and the United States. J Am Coll Surg 2006;202(1):45-54

23 Slim K, Panis Y, Chipponi J; Société Française de Chirurgie Digestive. Half of the current practice of gastrointestinal surgery is against the evidence: a survey of the French Society of Digestive Surgery. J Gastrointest Surg 2004;8(8):1079-1082

24 Walter CJ, Smith A, Guillou P. Perceptions of the application of fasttrack surgical principles by general surgeons. Ann R Coll Surg Engl 2006;88(2):191-195

25 Lassen K, Hannemann P, Ljungqvist O, et al; Enhanced Recovery After Surgery Group. Patterns in current perioperative practice: survey of colorectal surgeons in five northern European countries. BMJ 2005;330(7505):1420-1421

26 Delaney CP, Senagore AJ, Gerkin TM, et al. Association of surgical care practices with length of stay and use of clinical protocols after elective bowel resection: results of a national survey. Am J Surg 2010;199(3):299-304, discussion 304

27 Cabana MD, Rand CS, Powe NR, et al. Why don't physicians follow clinical practice guidelines? A framework for improvement. JAMA 1999;282(15):1458-1465

28 Andersen J, Hjort-Jakobsen D, Christiansen PS, Kehlet H. Readmission rates after a planned hospital stay of 2 versus 3 days in fasttrack colonic surgery. Br J Surg 2007;94(7):890-893

29 Wilmore DW, Kehlet $\mathrm{H}$. Management of patients in fast track surgery. BMJ 2001;322(7284):473-476

30 Chestovich PJ, Lin AY, Yoo J. Fast-track pathways in colorectal surgery. Surg Clin North Am 2013;93(1):21-32
31 Tonnesen H, Rosenberg J, Nielsen HJ, et al. Effect of preoperative abstinence on poor postoperative outcome in alcohol misusers: randomised controlled trial. BMJ 1999;318(7194):1311-1316

32 Tønnesen $\mathrm{H}$. Alcohol abuse and postoperative morbidity. Dan Med Bull 2003;50(2):139-160

33 Mills E, Eyawo O, Lockhart I, Kelly S, Wu P, Ebbert JO. Smoking cessation reduces postoperative complications: a systematic review and meta-analysis. Am J Med 2011;124(2):144-154, e8

34 Wong J, Lam DP, Abrishami A, Chan MT, Chung F. Short-term preoperative smoking cessation and postoperative complications: a systematic review and meta-analysis. Can J Anaesth 2012;59 (3):268-279

35 Sørensen LT. Wound healing and infection in surgery: the pathophysiological impact of smoking, smoking cessation, and nicotine replacement therapy: a systematic review. Ann Surg 2012;255 (6):1069-1079

36 Fleisher LA, Beckman JA, Brown KA, et al; American College of Cardiology Foundation/American Heart Association Task Force on Practice Guidelines; American Society of Echocardiography; American Society of Nuclear Cardiology; Heart Rhythm Society; Society of Cardiovascular Anesthesiologists; Society for Cardiovascular Angiography and Interventions; Society for Vascular Medicine; Society for Vascular Surgery. 2009 ACCF/AHA focused update on perioperative beta blockade incorporated into the ACC/AHA 2007 guidelines on perioperative cardiovascular evaluation and care for noncardiac surgery. J Am Coll Cardiol 2009;54(22): e13-e118

37 Chopra V, Eagle KA. Perioperative mischief: the price of academic misconduct. Am J Med 2012;125(10):953-955

38 Güenaga KF, Matos D, Wille-Jørgensen P. Mechanical bowel preparation for elective colorectal surgery. (Review) Cochrane Database Syst Rev 2011;(9):CD001544

39 Zerey M, Hawver LM, Awad Z, Stefanidis D, Richardson W, Fanelli RD; Members of the SAGES Guidelines Committee. SAGES evidence-based guidelines for the laparoscopic resection of curable colon and rectal cancer. Surg Endosc 2013;27(1):1-10

40 Bessey PQ. Metabolic Response to Critical Illness. New York, NY: Scientific American Inc; 1995

41 Minowada G, Welch WJ. Clinical implications of the stress response. J Clin Invest 1995;95:3-12

42 Desborough JP. The stress response to trauma and surgery. $\mathrm{Br}$ J Anaesth 2000;85(1):109-117

43 Blackburn GL. Metabolic considerations in management of surgical patients. Surg Clin North Am 2011;91(3):467-480

44 Kehlet H. Fast-track surgery-an update on physiological care principles to enhance recovery. Langenbecks Arch Surg 2011;396(5):585-590

45 Kleinman W, Mikhail MS, Murray MJ. Spinal, epidural and caudal blocks. In: Clinical Anesthesiology. 4th ed. New York, NY: McGrawHill; 2005

46 Wilmore DW. From Cuthbertson to fast-track surgery: 70 years of progress in reducing stress in surgical patients. Ann Surg 2002;236 (5):643-648

47 Senagore AJ, Delaney CP, Mekhail N, Dugan A, Fazio VW. Randomized clinical trial comparing epidural anaesthesia and patientcontrolled analgesia after laparoscopic segmental colectomy. Br J Surg 2003;90(10):1195-1199

48 Jørgensen H, Wetterslev J, Møiniche S, Dahl JB. Epidural local anaesthetics versus opioid-based analgesic regimens on postoperative gastrointestinal paralysis, PONV and pain after abdominal surgery. Cochrane Database Syst Rev 2000;(4):CD001893

49 Herroeder S, Pecher S, Schonherr ME, et al. Systemic lidocaine shortens length of hospital stay after colorectal surgery: a doubleblinded, randomized, placebo-controlled trial. Ann Surg 2007;246 (2):192-200

50 Rafi AN. Abdominal field block: a new approach via the lumbar triangle. Anesthesia 2001;56(10):1024-1026 
51 McDonnell JG, O’Donnell B, Curley G, Heffernan A, Power C, Laffey JG. The analgesic efficacy of transversus abdominus plane block after abdominal surgery: a prospective randomized controlled trial. Anesth Anal 2007;104(1):193-197

52 Delaney CP, Marcello PW, Sonoda T, Wise P, Bauer J, Techner L. Gastrointestinal recovery after laparoscopic colectomy: results of a prospective, observational, multicenter study. Surg Endosc 2010; 24(3):653-661

53 Delaney CP, Chang E, Senagore AJ, Broder M. Clinical outcomes and resource utilization associated with laparoscopic and open colectomy using a large national database. Ann Surg 2008;247(5): 819-824

54 Kuhry E, Schwenk WF, Gaupset R, Romild U, Bonjer HJ. Long-term results of laparoscopic colorectal cancer resection. Cochrane Database Syst Rev 2008;(2):CD003432

55 Schwenk WF, Haase O, Neudecker J, Müller JM. Short term benefits for laparoscopic colorectal resection. Cochrane Database Syst Rev 2005;(3):CD003145

56 Senagore AJ, Delaney CP. A critical analysis of laparoscopic colectomy at a single institution: lessons learned after 1000 cases. Am J Surg 2006;191(3):377-380

57 Delaney CP, Brady K, Woconish D, Parmar SP, Champagne BJ. Towards optimizing perioperative colorectal care: outcomes for 1,000 consecutive laparoscopic colon procedures using enhanced recovery pathways. Am J Surg 2012;203(3):353-355, discussion 355-356

58 Delaney CP. Outcome of discharge within 24 to 72 hours after laparoscopic colorectal surgery. Dis Colon Rectum 2008;51 (2):181-185

59 Poon JT, Fan JK, Lo OS, Law WL. Enhanced recovery program in laparoscopic colectomy for cancer. Int J Colorectal Dis 2011;26(1): 71-77

60 King PM, Blazeby JM, Ewings P, et al. Randomized clinical trial comparing laparoscopic and open surgery for colorectal cancer within an enhanced recovery program. Br J Surg 2006;2006 (93):300-308

61 Reurings JC, Spanjersberg WR, Oostvogel HJ, et al. A prospective cohort study to investigate cost-minimisation, of Traditional open, open fAst track recovery and laParoscopic fASt track multimodal management, for surgical patients with colon carcinomas (TAPAS study). BMC Surg 2010;10:18
62 Vlug MS, Wind J, Hollmann MW, et al; LAFA study group. Laparoscopy in combination with fast track multimodal management is the best perioperative strategy in patients undergoing colonic surgery: a randomized clinical trial (LAFA-study). Ann Surg 2011;254(6):868-875

63 Vlug MS, Bartels SA, Wind J, Ubbink DT, Hollmann MW, Bemelman WA; Collaborative LAFA Study Group. Which fast track elements predict early recovery after colon cancer surgery? Colorectal Dis 2012;14(8):1001-1008

64 Kennedy RH, Francis A, Dutton S, et al. EnROL: a multicentre randomised trial of conventional versus laparoscopic surgery for colorectal cancer within an enhanced recovery programme. BMC Cancer 2012;12:181

65 Trastulli S, Cirocchi R, Lisorti C, et al. Laparoscopic vs open resection for rectal cancer: a meta-analysis of randomized clinical trials. Colorectal Dis 2012;14(6):e297-e296

66 Nygren J, Thacker J, Carli F, et al. Guidelines for perioperative care in rectal/pelvic surgery: Enhanced Recovery After Surgery (ERAS®) Society recommendations. World J Surg 2013;37 (2):285-305

67 Senagore AJ. Pathogenesis and clinical and economic consequences of postoperative ileus. Am J Health Syst Pharm 2007;64(20, Suppl 13):S3-S7

68 Livingston EH, Passaro EPJ Jr. Postoperative ileus. Dig Dis Sci 1990;35(1):121-132

69 Council PIM. Available at: www.clinicalwebcasts.com/PIMC.htm. Accessed October 10, 2012.

70 Goldstein JL, Matuszewski KA, Delaney CP, et al. Inpatient economic burden of postoperative ileus associated with abdominal surgery in the United States. Pharmacy Therapeutics J 2007;32(2):82-90

71 Sinatra RS, Larach S, Ramamoorthy S. Surgeon's Guide to Postsurgical Pain Management: Colorectal and Abdominal Surgery. West Islip, NY: Professional Communications; 2012

72 Kehlet H, Slim K. The future of fast-track surgery. Br J Surg 2012;99 (8):1025-1026

73 Bardram L, Funch-Jensen P, Kehlet H. Rapid rehabilitation in elderly patients after laparoscopic colonic resection. Br J Surg 2000;81(11):1540-1545

74 Basse L, Jakobsen DH, Bardram L, et al. Functional recovery after open versus laparoscopic colonic resection: a randomized, blinded study. Ann Surg 2005;241(3):416-423 